\title{
Integration of gastronomy and physics for innovation
}

\author{
Erik van der Linden
}

\begin{abstract}
Integration of physics with gastronomy can yield innovations in an efficient manner. An important element of this integration is the structure of food. The creation of food recipes often deals with designing new structures and a clear understanding of how food structure influences food properties is necessary. The physics that is required for this understanding can be demonstrated by considering the case of gelatin. A Master of Science (MSc) specialization is described, which addresses the integration of physics with gastronomy in an educational setting at Wageningen University, The Netherlands.
\end{abstract}

\section{Introduction}

At fast food counters, customers can choose between eating the meal at that place or to take it out. This food is 'ready to eat'. Although this food serves its purpose of providing nourishment, it does not usually create a high level of enjoyment. The latter is more a signature of what is currently recognized as 'gastronomy' and is practised in more sophisticated restaurants. Gastronomy is sometimes defined as 'the art of enjoyably eating and drinking'. This is usually achieved by stimulating different senses all at the same time, and/or stimulating one of the senses at different rates and levels.

Historically, the first phase of gastronomy evolution has been the creation of classical recipes over many previous centuries, which, at their beginning, may be referred to as innovations. The second phase has been the improvement of classical recipes. The third phase has been the formation of new and creative recipes by means of using ingredients and/or techniques that are novel, at least to the restaurant scene. It is these recipes that produce culinary innovation and trigger the overall sensory perception.

This overall sensory perception is composed of contributions from different senses. These contributions are interrelated, that is, changing one contribution can affect other contributions. In order to be efficient in achieving an overall positive sensory innovation, by means of using

Correspondence: erik.vanderlinden@wur.nl Laboratory of Physics and Physical Chemistry of Foods, Wageningen University, Wageningen, The Netherlands a new ingredient and/or technique, one needs an integrated approach regarding contributions from the different senses. Such integration should avoid that optimization if one contribution is detrimental to another. The integration is facilitated by physics, since most of the sensory contributions are physics-related: texture (relates to mechanics), fracture and breakdown during mastication (relates to sound), color (relates to optics), and smell (relates to volatile release and transport). The integration of physics with gastronomy can therefore yield innovations in an efficient manner.

The above provides an example of the general argument, originally presented by Donald Stokes [1], and suggests that the chance of innovation is highest when one integrates research that is curiosity-driven (in this case physics) with research that is application-driven (in this case gastronomy). It is important to note that curiosity-driven research also requires a continuous effort of development.

\section{The importance of structure for integration}

For food applications, from a physics point of view, one needs to understand how macroscopic physical food properties relate to molecular properties and interactions of the ingredients, as a function of the parameters of ingredient concentration, ingredient type, energy input, temperature and time. The values of these parameters are reflected by the food's microstructure. This structure allows an understanding of how macroscopic and molecular properties are related. Changing the preparation conditions will change the position of the 
diagram in Figure 1 and therefore the structure can change as well.

The design of new structures can allow the current physical theories to be tested and may yield new physical understanding. It may also yield new materials with new applications. This means that one can end up with different and even new structures when taking different trajectories through the diagram. In other words, taking novel paths through the diagram (such as novel ingredients and novel techniques or processes) can allow the design of new structures. The new structures allow the testing of existing physics and may even provide new physics. At the same time, designing new structures implies new material properties, functions and applications.

The above is an illustration of the overarching vision of the author's chair group of Physics and Physical Chemistry of Foods at Wageningen University, The Netherlands. According to this vision, an integration of physics with structure design and application-driven research (in this case gastronomy) yields innovations in an efficient manner [2]. Similarly, according to this same vision, an educational effort integrating science and application (in the current case of physics and gastronomy) may form the basis of an innovation competency for our graduates. A recently established Master of Science (MSc) specialization at Wageningen University, with interactions with the Rijn IJssel school for chefs in Wageningen, has been developed along these lines [3].

\section{An example of physics relevant to gelatin}

Gelatin elasticity, in terms of the characteristics of the gelatin microstructure, will be described in order to appreciate the depth of the physics required. Certain aspects of the physics have been developed only ten years ago. The account in this section has been addressed earlier $[4,5]$ and will be briefly summarized.

A gelatin gel consists of triple helices connected by coillike structures. Professor Djabourov and her group [6] proved that the type of gelatin does not matter for the elasticity, only the concentration of helices. They also demonstrate that the elasticity at low concentrations follows a scaling with an exponent of scalar percolation and with a critical concentration. The critical concentration is related to the persistence length, $L_{p}$, and hence stiffness of the helices [4]. A percolating (network) structure arises when there are about three contacts per length $\mathrm{L}_{\mathrm{p}}$. The stiffness of the helix also determines the initial elasticity of the gel [5]. At higher concentrations, a deflection length, $L_{d}$, needs to be introduced, which is the length over which the helix is stiff when confined within a tube of thickness D. This second characteristic length scale, $\mathrm{L}_{\mathrm{d}}$, in the system was introduced by Odijk [7]. The confinement of the helix by means of $\mathrm{D}$ is determined by the concentration of helices. The higher the concentration, the smaller $\mathrm{D}$ and the smaller $\mathrm{L}_{\mathrm{d}}$. The exact dependency of $L_{d}$ on the concentration of helices is known [7]. The contribution to the elastic component of the shear modulus, G, due to the deflection length at high concentration, is given by:

$$
\mathrm{G}^{\prime}=\mathrm{n} * \mathrm{k}_{\mathrm{B}} \mathrm{T} *\left(\mathrm{~L} / \mathrm{L}_{\mathrm{d}}\right)
$$

where $n$ denotes the number of helices per volume, $k_{B} T$ the thermal energy and $L$ the contour length of the helices. Substituting the dependency of $L_{d}$ on concentration allows deducing the elasticity contribution at high concentrations. There is one parameter that needs to be fitted using the high concentration data and that is the pre-factor (of order unity) in the scaling relation for high concentration. Finally, the total elasticity can be viewed as the sum contribution of two networks in series. The overall G' fits the elasticity of the gelatin perfectly [4].

The analysis shows that the deflection length is a characteristic length scale arising from the helix confinement at higher helix concentrations. In the end, only the temperature and the persistence length of the helices are

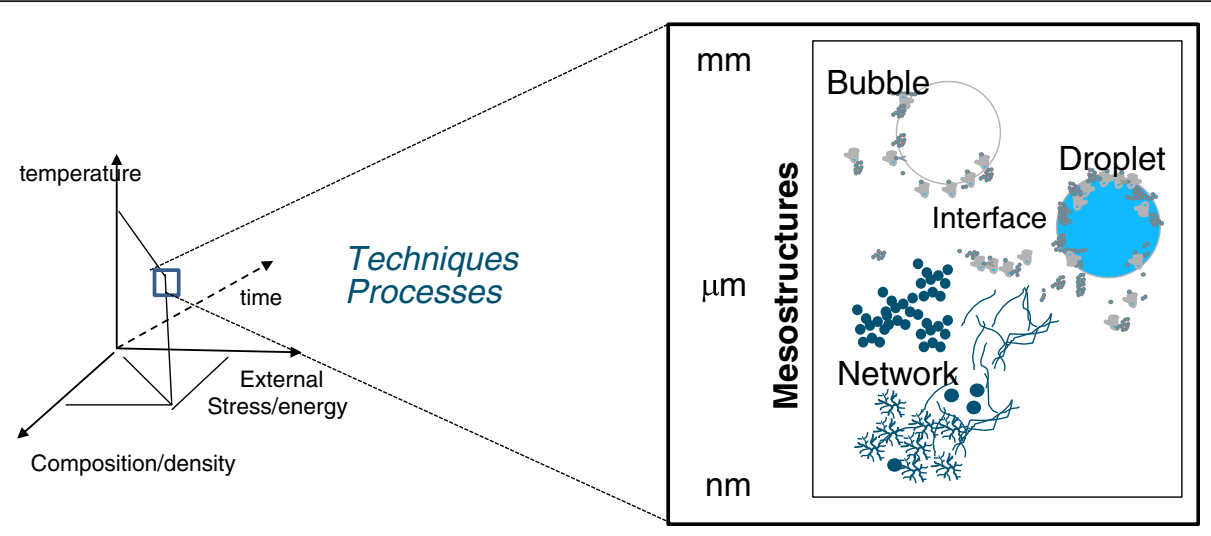

Figure $1 \mathrm{~A}$ trajectory through the parameter space, as initiated by techniques and processes, can change the according food structure. 
the sole first parameters for the elasticity of the gelatin gel. This insight is beautiful by its simplicity, while the physics behind it remains complex, including the adaptation of helix dynamics to helix concentration. The simplification can help in efficient structure design.

\section{Integration for innovation for educational purposes}

In a recently established MSc specialization at Wageningen University, two course modules are based on integrating physics (and chemistry) with gastronomy. Both modules address physics and chemistry knowledge, and practical assignments challenge the creativity of the students and their knowledge of physics and chemistry with relevance to gastronomy. The practical assignments are performed at the Rijn IJssel school for chefs in Wageningen, which provides the necessary infrastructure. It also involves teachers from that school to cover the technical skills and knowledge from a chef's perspective. It exposes the students to real food systems instead of only model systems. Innovative recipes are developed and the best ones are to be incorporated into a book, which will also address recipe innovation as well as its scientific background. These results may also benefit other food professionals.

\section{Conclusions and outlook}

The opinion that the integration of physics and gastronomy can lead to innovations in an efficient manner is based on the general argument presented by Stokes [1]. Food structure and its design provide an important element in this integration. Physics is sometimes able to simplify matters considerably, as demonstrated in the case of gelatin, where the persistence length of the helices and the temperature are the only structure parameters necessary to explain the elasticity of a gelatin gel. The integration of physics and gastronomy has been incorporated in an MSc specialization and it is expected that this will help MSc graduates to apply the integration in their future careers of innovation.

\section{Competing interests}

The author declares that he has no competing interests.

Received: 9 November 2012 Accepted: 9 November 2012 Published: 9 January 2013

\section{References}

1. Stokes DE: Pasteur's Quadrant: Basic Science and Technological Innovation. Washington DC: Brookings Institution Press; 1997.

2. Physics and Physical Chemistry of Foods chair group at Wageningen University, The Netherlands: http://www.wageningenur.nl/fph.

3. MSc Gastronomy at Wageningen University, The Netherlands: http://www. wageningenur.nl/en/Education-Programmes/Prospective-Master-Students/ MSc-programmes/MSc-Food-Technology/Specialisations/Gastronomy.htm.

4. van der Linden E, Parker A: Elasticity due to semiflexible protein assemblies near the critical gel concentration and beyond. Langmuir 2005, 21:9792-9794.

5. van der Linden E: From peptides and proteins to micro-structure mechanics and rheological properties of fibril systems. Food Hydrocolloids 2012, 26/2:421-426.
6. Joly-Duhamel C, Hellio D, Ajdari A, Djabourov M: All Gelatin Networks: 2. The Master Curve for Elasticity. Langmuir 2002, 18:7158-7166.

7. Odijk T: The statistics and dynamics of confined or entangled stiff polymers. Macromolecules 1983, 16:1340-1344.

doi:10.1186/2044-7248-2-11

Cite this article as: van der Linden: Integration of gastronomy and physics for innovation. Flavour 2013 2:11.

\section{Submit your next manuscript to BioMed Central and take full advantage of:}

- Convenient online submission

- Thorough peer review

- No space constraints or color figure charges

- Immediate publication on acceptance

- Inclusion in PubMed, CAS, Scopus and Google Scholar

- Research which is freely available for redistribution 